\title{
Resonant Energy Transfer of Triplet Excitons from Pentacene to PbSe Nanocrystals
}

Maxim Tabachnyk, Bruno Ehrler, Simon Gélinas, Marcus L. Böhm, Brian J. Walker, Kevin P. Musselman, Neil C. Greenham, Richard H. Friend and Akshay Rao

Cavendish Laboratory, J.J. Thomson Avenue, University of Cambridge, Cambridge CB3 OHE, UK 


\begin{abstract}
The efficient transfer of energy between organic and inorganic semiconductors is a widely sought after property, but has to date been limited to the transfer of spin singlet excitons. Here we report efficient resonant-energy transfer of molecular spin triplet excitons from organic semiconductors to inorganic semiconductors. We use ultrafast optical absorption spectroscopy to track the dynamics of triplets, generated in pentacene via singlet exciton fission, at the interface with lead selenide $(\mathrm{PbSe})$ nanocrystals. We show that triplets transfer to $\mathrm{PbSe}$ rapidly $(<1 \mathrm{ps})$ and efficiently, with 1.9 triplets transferred for every photon absorbed in pentacene, but only when the bandgap of the nanocrystals is close to resonance $( \pm 0.2 \mathrm{eV})$ with the triplet energy. Following triplet transfer, the excitation can undergo either charge separation, allowing photovoltaic operation, or radiative recombination in the nanocrystal, enabling luminescent harvesting of triplet exciton energy in light emitting structures.
\end{abstract}




\section{Main Text}

The coupling of organic and inorganic semiconductors, enabling efficient energy transfer between them, is a long standing goal ${ }^{1-4}$, with applications in photovoltaics (PVs) ${ }^{1,5}$, light emitting-diodes (LEDs) ${ }^{6}$ and sensors ${ }^{7,8}$. Within organic semiconductors, the low dielectric constant leads to the formation of strongly bound excitons, which can be either spin-singlet (spin 0) or spin-triplet (spin 1). To date, efforts to couple organic and inorganic semiconductors have focussed on the transfer of singlet excitons via Förster resonance energy transfer (FRET) ${ }^{2-4}$. In contrast, the transfer of triplet excitons via FRET is spin forbidden ${ }^{9}$. But triplet excitons possess properties such as long lifetimes, up to several ms, and diffusion lengths, up to several $\mu \mathrm{m}^{10}$, which makes them very attractive for use within long-range energy transfer schemes ${ }^{1,4}$. Here we establish the conditions under which molecular triplet excitons can undergo efficient transfer into inorganic semiconductors.

Singlet exciton fission (SF) is a process in organic semiconductors, by which a single photogenerated spin-singlet exciton is converted to two spin-triplet excitons on nearby chromophores, Figure $1 \mathrm{a}^{11}$. As the process is spin allowed, it can occur on sub $100 \mathrm{fs}$ timescales with an efficiency of $200 \%{ }^{12,13}$, when the energetics of the system are favourable, i.e. the energy of the spin-singlet exciton is greater than or equal to twice the energy of the spin-triplet exciton. $\mathrm{SF}$ is a promising route to overcome the Shockley-Queisser limit in single-junction photovoltaics $^{14}$, if a SF material could be suitably combined with a low-bandgap inorganic semiconductor. In this configuration, illustrated in Figure 1b, the low-bandgap semiconductor generates one electron-hole pair for each low-energy photon absorbed, while the SF material 
generates two triplet excitons for each high-energy photon absorbed. By distributing the energy of high-energy photons into two excitations, SF could allow solar cells to overcome the otherwise dominant thermalisation losses. Ideally, the energy of the triplet excitons could be directly transferred into the inorganic semiconductor, for which charge generation and collection are already optimized. This approach would allow the SF material to act as an energy-funnelling layer on top of a conventional PV, rather than being an active part of the circuit. The potential benefits of such an approach were recognised over thirty years ago in the pioneering work of Dexter ${ }^{1}$, and calculations show that this would raise the theoretical maximum power conversion efficiency for single-junction photovoltaics from $34 \%$ to $44 \%^{11,15}$. However, the transfer of molecular triplet excitons from organic to inorganic semiconductors has never been demonstrated directly.

Pentacene $(\mathrm{Pc})$ is a model system for singlet exciton fission. Previous transient optical absorption (TA) measurements on Pc determined a fission rate of $80 \mathrm{fs}$, outcompeting alternative decay mechanisms ${ }^{12,13,16}$, such as electron transfer or singlet energy transfer via FRET. The fissiongenerated triplets can be efficiently dissociated at a heterojunction using the fullerene $\mathrm{C}_{60}$ as the $\operatorname{acceptor}^{17}$, allowing for external quantum efficiencies (EQE) of $126 \%$, the highest for any photovoltaic technology to date ${ }^{18,19}$. Devices combining Pc with low-bandgap PbSe nanocrystals as the acceptor have also been recently demonstrated ${ }^{20}$. There are two possible pathways for charge generation at such organic inorganic interfaces, as shown in Figure 1c. The first is electron transfer from $\mathrm{Pc}$ to $\mathrm{PbSe}$. The second is energy transfer of triplet excitons into $\mathrm{PbSe}$, followed by back-transfer of holes into Pc. For this system, the HOMO of Pc is at $-5.05 \mathrm{eV}$ and the valence band of the PbSe nanocrystals is at -5.1 , allowing hole transfer from PbSe to $\mathrm{Pc}$ (see S16 for details). 
To investigate the dynamics of Pc triplet excitons at the interface with $\mathrm{PbSe}$, we performed TA measurements on thin $\mathrm{PbSe} / \mathrm{Pc}$ bilayers, consisting of 1-2 monolayers of spin-coated $\mathrm{PbSe}$, onto which $5 \mathrm{~nm}$ of Pc (3 molecular layers) was evaporated. AFM, SEM and absorption data are provided in the SI (S2-4). The thinness of the pentacene layer ensures that all triplet excitons are generated close to the interface with $\mathrm{PbSe}$. This allows us to probe interfacial dynamics, which are normally masked by bulk diffusion processes. The samples were investigated with femtosecond (fs) TA spectroscopy, using a narrowband pump pulse centred at $550 \mathrm{~nm}$ and broadband probe pulses. In order to amplify the signal from the extremely thin layers, we use an optical cavity, which allows for multiple passes of collinear pump and probe beams through the sample $^{21}$ (See Methods). A series of PbSe nanocrystals, with benzenedithiol (BDT) as the ligand and bandgap energies between 0.67 and $1.61 \mathrm{eV}$ were compared against Pc (See SI).

Figure 1d compares the TA kinetics, at $670 \mathrm{~nm}$, in pristine $\mathrm{Pc}$ and $\mathrm{Pc} / \mathrm{PbSe}$ bilayers with nanocrystals of varying bandgap. $670 \mathrm{~nm}$ is the position of the peak of the Pc ground state bleach $(\mathrm{GSB})^{16}$. The lowest lying molecular triplet exciton $\left(\mathrm{T}_{1}\right)$ in Pc has been reported to lie close to $0.86 \mathrm{eV}^{20,22}$. The two bilayers with nanocrystals of bandgap far below $(0.67 \mathrm{eV})$ and above (1.61 eV) this energy, show almost identical kinetics to the pristine Pc film. In contrast, the bilayer with nanocrystals of bandgap $0.78 \mathrm{eV}$, which is close to resonance with $\mathrm{T}_{1}$, shows a significant drop in signal within the first 2 ps followed by a revival at later times. As we develop below, this kinetic behaviour is due to energy transfer, de-exciting the chromophore, followed by back charge transfer, re-exciting the chromophore.

However, the presence of excited state signals from the PbSe also needs to be taken into account, before we can quantify the populations of excited state species. Figure 2a shows TA spectra of the $\mathrm{Pc} / \mathrm{PbSe}(0.78 \mathrm{eV})$ bilayer (full set of TA data for other nanocrystal films are provided in the 
SI, S5-11). In order to extract the individual spectra and population kinetics of the various excited state species we use a genetic algorithm ${ }^{23,24}$ (see Methods). The data in Figure 2a can be decomposed into three excited state species, with spectra shown by the solid lines in Figure $2 \mathrm{~b}$ and corresponding population kinetics shown in Figure 2c. One component (solid blue) matches the signal expected for Pc, as demonstrated by the agreement with the spectrum of a pristine Pc film (dashed blue spectra). We note that after the ultrafast fission process ( $80 \mathrm{fs}$ ), there is no spectral evolution in the signal from $\mathrm{Pc}^{13,16}$. Thus, in the experiments presented here, with a time resolution $>300 \mathrm{fs}$, only a single spectrum is expected for Pc. The nanocrystals show two distinct spectra in this region (solid black and solid red), associated with relaxation from higher to lower energy excited states $^{25}$, as demonstrated by the early and later time signals from pristine PbSe films (dashed spectra).

We now turn to the kinetics extracted by the genetic algorithm, shown in Figure 2c, which represent the weight of the spectral component of an excited state species in the whole data set, rather than kinetics at a particular spectral point. We observe that the Pc component in the $\mathrm{Pc} / \mathrm{PbSe}(0.78 \mathrm{eV})$ film (solid blue) drops faster than in pristine Pc films (dashed blue), over the initial 0.3-3 ps, but subsequently rebounds (after $5 \mathrm{ps}$ ), and at later times (>30 ps) has a larger signal than pristine Pc films. The population in the Pc, at the earliest times we can observe (300 fs) consists entirely of triplet excitons, due to the ultrafast ( $80 \mathrm{fs}$ ) fission process ${ }^{13}$. Thus the reduction in signal between $0.2-3$ ps represents a loss of triplet excitons. The increase in signal at later times is consistent with hole-transfer from the PbSe to Pc. The rise of the lower-energy excited state component of $\mathrm{PbSe}$ (black curve) is associated with the reduction of the higherenergy PbSe excited state component (red curve), as well as the loss of triplets in Pc. This suggests that both processes could populate the lower-energy PbSe excited state. 
Lastly, we observe a blue-shifting in the GSB peaks of the PbSe nanocrystals, figure $2 \mathrm{~d}$. We consider this shift to be caused by charge transfer across the Pc/PbSe interface, which sets up microscopic electric fields, causing a Stark shift of the transition energy of species near the interface $^{17}$. This result in a derivative-like feature at the red-edge of the absorption feature, termed electroabsorption, which blue-shifts the GSB peak, as has been discussed in detail previously ${ }^{17,24}$ and been reported for $\mathrm{PbSe}^{26}$. The blue-shift of the GSB occurs over tens of ps, and hence, the associated charge-transfer process occurs over tens of ps. This timescale is consistent with the rebound in the Pc component, confirming the assignment of this rise to hole back transfer from the PbSe. No electroabsorption features or shifts in GSB were observed for pristine $\mathrm{PbSe}$ layers or $\mathrm{Pc} / \mathrm{PbSe}$ bilayers in which the triplet transfer was not observed (see $\mathrm{SI}$ ). Thus, the model that emerges from the data is one of forward triplet transfer from $\mathrm{Pc}$ to $\mathrm{PbSe}$ followed by back hole transfer from PbSe to Pc.

Figure 3a shows normalized kinetics, extracted via the GA, of the Pc component in both pristine a Pc film and $\mathrm{Pc} / \mathrm{PbSe}$ bilayers with varying nanocrystal bandgaps. Figure $3 \mathrm{~b}$ shows the corresponding $\mathrm{PbSe}$ component in both pristine nanocrystal films and $\mathrm{Pc} / \mathrm{PbSe}$ bilayers. The initial triplet transfer and subsequent hole transfer are observed only with nanocrystal bandgap of $0.78 \mathrm{eV}$, Figure 3a. In the other two cases, for nanocrystals of bandgap much higher or lower than the Pc triplet energy $(0.86 \mathrm{eV})$, the kinetics are almost identical to pristine pentacene films, indicting very little or no triplet transfer. Turning to the PbSe component, Figure $3 \mathrm{~b}$, for the 0.67 $\mathrm{eV}$ nanocrystals, there is only a small difference between pristine $\mathrm{PbSe}$ (dashed red) and $\mathrm{Pc} / \mathrm{PbSe}$ (solid red), suggesting that triplet injection from Pc is inefficient. However, for the $0.78 \mathrm{eV}$ nanocrystals, there is a large enhancement in signal for the $\mathrm{Pc} / \mathrm{PbSe}$ sample (solid blue) in comparison to the pristine $\mathrm{PbSe}$ (dashed blue). The signal peaks at a later time, 4-5 ps, consistent 
with the timescale for triplet transfer (Figure 3a). This shows that the increase in signal is due to efficient transfer of triplets to the PbSe. The population is also found to be longer-lived, consistent with the formation of longer-lived charges following back hole-transfer.

Figure $3 c$ illustrates the evolution of the $\mathrm{Pc}$ component in $\mathrm{Pc} / \mathrm{PbSe}(0.78 \mathrm{eV})$ bilayers with nanocrystal ligands of increasing length, benzenedithiol (BDT, 0.7nm), octanedithiol (ODT, $1.1 \mathrm{~nm}$ ) and oleic acid (OA, 2.2nm) (see SI, S10-12 for TA spectra and GA decomposition). We observe a more pronounced dip in the Pc component, corresponding to an increase of the triplet transfer efficiency with shorter ligands. Also, no significant triplet transfer in observed via monitoring with PbSe component for OA treated nanocrystals (see SI, S12).

Taken together, we find that efficient triplet transfer from Pc is only possible to nanocrystals with bandgap of $0.78 \mathrm{eV}$ or $0.93 \mathrm{eV}$ (see SI, S5-9), i.e. nanocrystals whose bandgap lie within a narrow range (less than $\pm 0.2 \mathrm{eV}$ ) of the Pc triplet energy $(0.86 \mathrm{eV})$, and also only when the nanocrystals have short ligands. The narrow energy range in which triplet transfer occurs efficiently indicates the importance of the overlap of the density of states of donor and acceptor, while the ligand length dependence points to the importance of wavefunction overlap.

The coupling integral for the energy transfer process contains contributions both from the Coulomb interaction and exchange interaction ${ }^{9,10}$. The negligible oscillator strength of the $\mathrm{S}_{0} \rightarrow \mathrm{T}_{1}$ transition for Pc means that the Coulomb interaction plays no significant role in the process. For the exchange interaction, Dexter ${ }^{27}$ derived that

$$
k \sim e^{-\frac{2 R}{L} J}
$$


where, $\mathrm{k}$ is the rate of transfer, $\mathrm{L}$ is the orbital radius of donor and acceptors site, $\mathrm{R}$ is the separation between them and $\mathrm{J}$ is the normalised spectral overlap between donor emission and acceptor absorption. Importantly, $\mathrm{J}$ is independent of the oscillator strengths of the optical transitions. Thus, triplet transfer would only be efficient to nanocrystals whose lowest-energy absorption feature, which has a large density of states, overlapped with the $S_{0} \rightarrow T_{1}$ transition for Pc, at about $0.8 \mathrm{eV}$. The width of the lowest-energy absorption feature for the nanocrystal studied here is about $0.15 \mathrm{eV}$ (see SI, S2) which corresponds well with the narrow range in which triplet transfer is observed, less than $\pm 0.2 \mathrm{eV}$.

We note that Dexter transfer normally occurs on very short length scales $(<10 \mathrm{~A})$, due to the requirement for orbital overlap 9 . The presence of ligands on the nanocrystals would at first glance preclude the possibility of transfer, however, it is clearly observed in the data presented above. This may be due to incomplete surface coverage of ligands that allows Pc molecules sufficient room to have orbital overlap with the PbSe (See S12). We also note, that electronic structure calculations for small-bandgap $\mathrm{PbS}$ show a wide separation in energy between the first excited state at $0.68 \mathrm{eV}$ and the second excited state at $0.96 \mathrm{eV}^{28}$. This could mean that the Pc triplet energy lies between the first and second excited states of the $0.67 \mathrm{eV}$ nanocrystal, overlapping with a region in which there are very few states available in the nanocrystal, leading to inefficient triplet transfer. Further experimental and theoretical work is called for to fully elucidate the mechanism and materials properties governing the triplet transfer process at such organic-inorganic interfaces.

For the $\mathrm{Pc} / \mathrm{PbSe}$ system studied here, triplet transfer can be followed by back hole transfer over tens of ps as seen in Figure 2 and 3 and discussed above. But a fraction of the excitations may not undergo hole-transfer and recombine within the $\mathrm{PbSe}^{29}$. Also at later times electron-hole 
recombination, of states previously separated across the interface, will occur. Both cases allow for radiative recombination and hence enhanced emission from the $\mathrm{PbSe}$, whenever triplet transfer is possible. Figure 4a shows the enhancement in PL from the PbSe nanocrystals, for a $\mathrm{Pc} / \mathrm{PbSe}(0.93 \mathrm{eV})$ bilayer in comparison to a PbSe $(0.93 \mathrm{eV})$ film. No change in relative PL is seen when exciting at 780 and $808 \mathrm{~nm}$, i.e. below the bandgap of Pc, but enhanced PL is seen when exciting above the $\mathrm{Pb}$ bandgap at 450, 532, 637 and $650 \mathrm{~nm}$ (See S13 for details). The enhanced PL is correlated with the Pc absorption (dashed line), confirming that it arises due to triplet energy transfer from the Pc. No PL enhancement arising due to excitation of Pc was observed for $\mathrm{Pc} / \mathrm{PbSe}$ samples where the nanocrystal bandgap blocked triplet transfer (see SI, S13), which further demonstrates the enhancement does not arise from early time (sub 100 fs) transfer of singlet excitons via FRET.

The PL of the $\mathrm{Pc} / \mathrm{PbSe}(0.93 \mathrm{eV})$ bilayer was found to be independent of an applied external magnetic field when exciting at $808 \mathrm{~nm}$, i.e. directly exciting the nanocrystals. However, when exciting the pentacene at $637 \mathrm{~nm}$ the PL decreased with increasing magnetic field, with a decrease of $4 \%$ at $800 \mathrm{mT}$ (see SI, S14). This magnetic field response is characteristic of singlet fission and has been previously observed, for instance in photocurrent from $\mathrm{Pc}$ devices ${ }^{18}$ and also in the PL from Pc single crystals, where a $2 \%$ increase in emission from singlets was found under applied magnetic field ${ }^{30}$. This corresponds well with the $4 \%$ decrease in PL we observe, since each singlet is converted into two triplets and transferred into $\mathrm{PbSe}$. This provides further proof that the enhanced PL from PbSe arises due to triplet transfer from Pc.

Near the peak of the Pc absorption, at $650 \mathrm{~nm}$, the $\mathrm{Pc} / \mathrm{PbSe}(0.93 \mathrm{eV})$ bilayer absorbs $44 \%$ more light than the PbSe $(0.93 \mathrm{eV})$ film (see SI, S15). Crucially, at this wavelength the PL is enhanced by $85 \%$ (Figure $4 \mathrm{a}$ ). This means that for every photon absorbed by Pc 1.9 excitations contribute 
to the PL. As shown above, triplet transfer is the dominant process in the bilayers. Assuming that singlet fission proceeds with a $200 \%$ yield of triplets, this implies a minimum triplet transfer efficiency of $95 \%$. We note, that by suitable choice of SF material and inorganic acceptor, it would be possible to arrange energetics such that back hole transfer is blocked, allowing only for triplet transfer.

In conclusion, we have reported the first demonstration of triplet energy transfer from organic to inorganic semiconductors. Our studies of the photophysics of thin bilayer samples of pentacene/PbSe nanocrystals demonstrate that triplet energy transfer from pentacene to $\mathrm{PbSe}$ is efficient only when the nanocrystal bandgap is resonant with the molecular triplet energy. This result opens new avenues to couple organic and inorganic semiconductors and new possibilities for devices. For instance, to harness non-radiative triplet excitons generated via electrical injection of charges in to organic LEDs. The triplets could be harvested via transfer into inorganic nanocrystals where the electron-hole pair could recombine radiatively, allowing for white-light emission without the need for phosphorescent molecules. As demonstrated here, this process can also be used to harness triplet excitons generated via singlet exciton fission, allowing the energy of the triplets to be directly funnelled in to conventional inorganic solar cells. This offers a very promising method to overcome the Shockley-Queisser limit. 


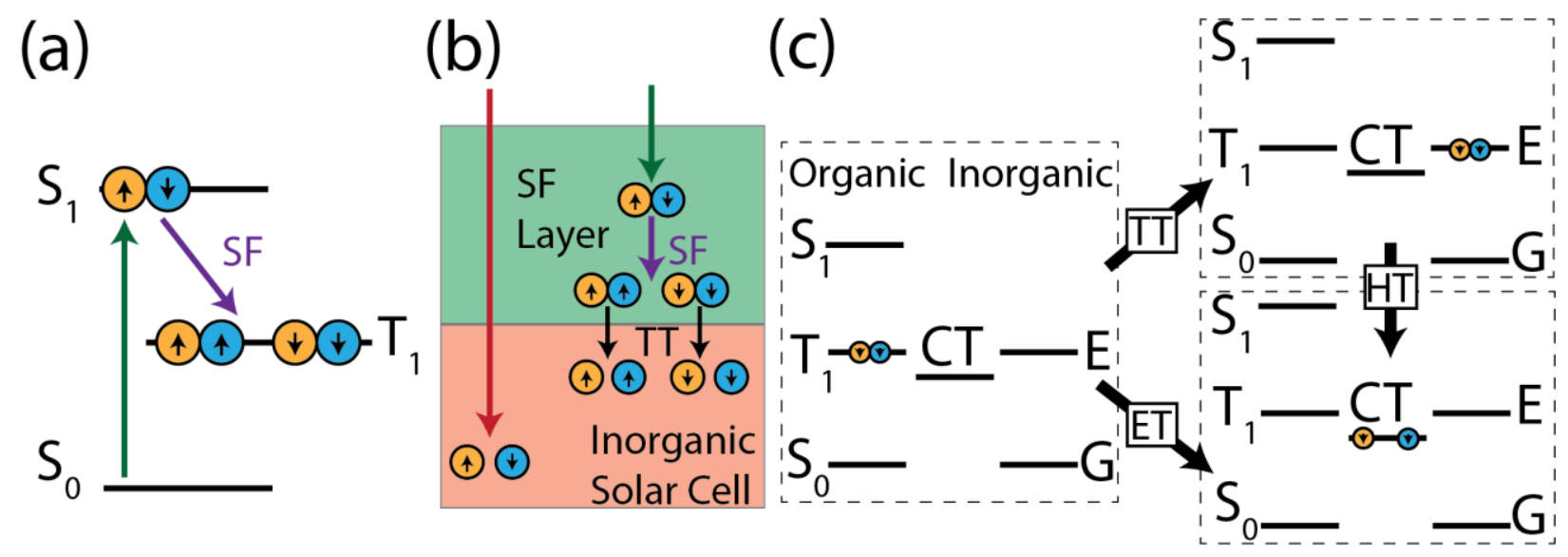

(d)

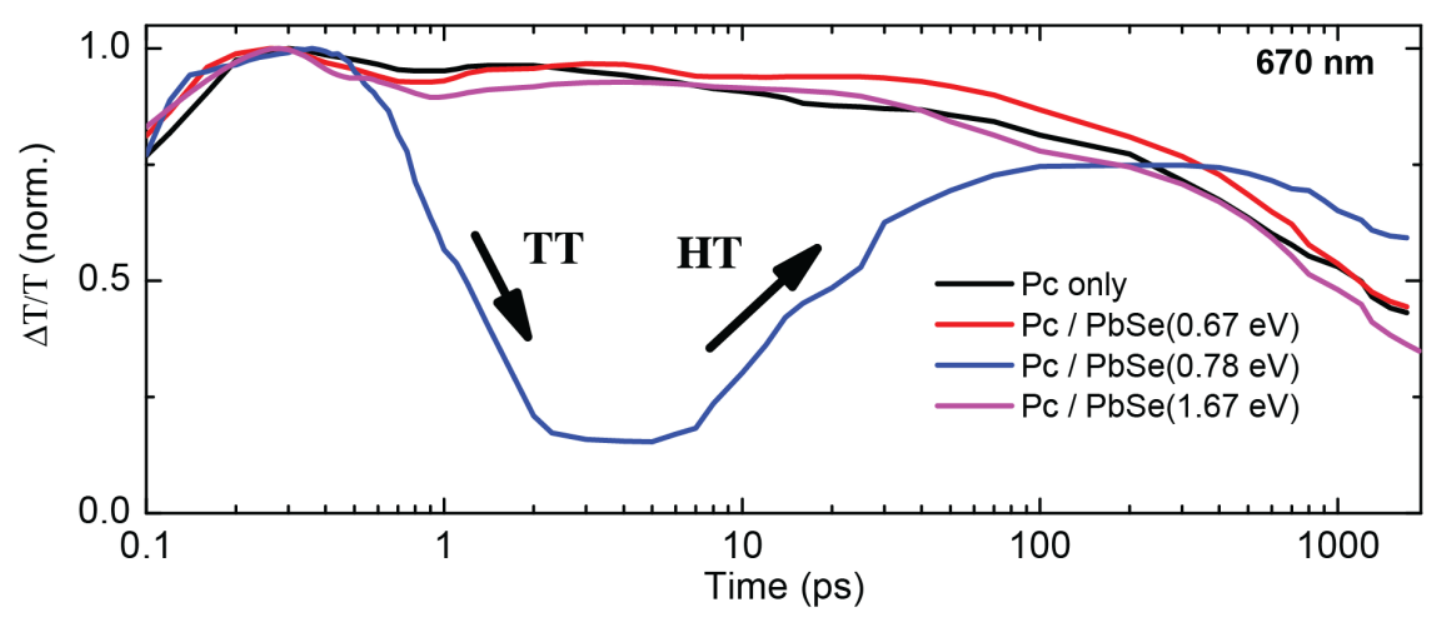

Figure 1: Singlet exciton fission and triplet transfer (a) Singlet exciton fission (SF) in pentacene $(\mathrm{Pc})$. Photo-generated singlet excitons $\left(\mathrm{S}_{1}\right)$ in $\mathrm{Pc}$ undergo fission into two triplet excitons $\left(\mathrm{T}_{1}\right)$ within 80 fs. (b) illustrates how inorganic solar cells can be singlet fission sensitized using triplet transfer from a thin organic SF layer. The organic layer absorbs highenergy photons, which generate singlet excitons and undergo singlet fission to form triplet excitons. The triplet excitons are then transferred into the inorganic layer where charges can easily be separated and extracted at electrodes. Simultaneously the inorganic harvests low-energy photons. (c) The possible processes a triplet exciton undergoes at an organic/inorganic interface; direct electron transfer (ET) or triplet transfer (TT). CT is the charge transfer state with hole on the organic and electron on the inorganic, $\mathrm{G}$ is the ground state of the inorganic and $\mathrm{E}$ the excited state. TT can be followed by hole transfer (HT) back into Pc to obtain a CT state. (d) compares the kinetics at the main Pc GSB at $670 \mathrm{~nm}$ in pristine Pc and bilayers with lead selenide (PbSe) nanocrystals of different bandgaps (in brackets). The bilayer where the PbSe bandgap is close to resonance with $\mathrm{T}_{1}(0.86 \mathrm{eV})$ shows a drop and revival, indicating TT followed by HT. 

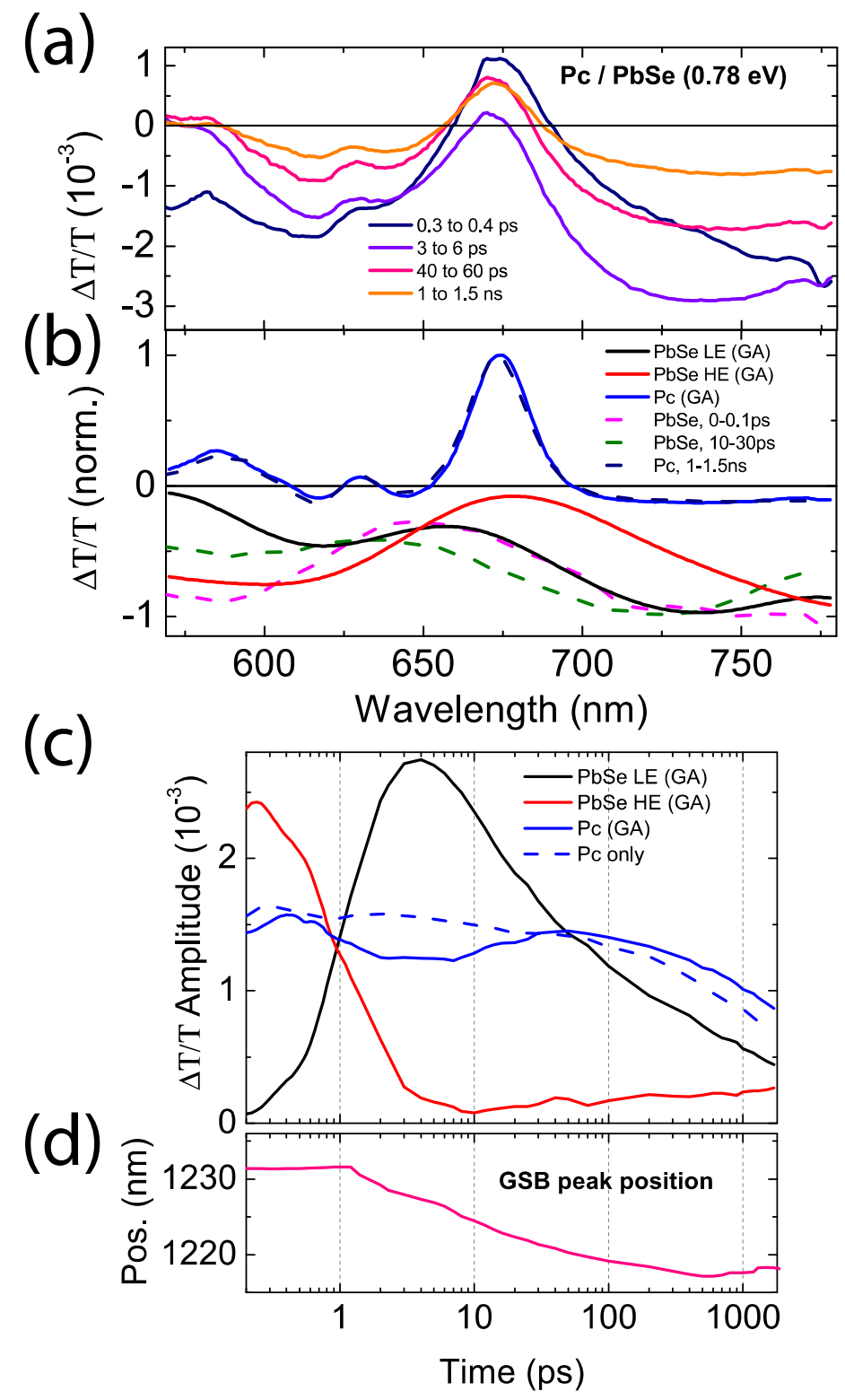

Figure 2: Numerical decomposition of transient absorption (TA) data. (a) shows the TA spectra of $\mathrm{Pc} / \mathrm{PbSe}(0.78 \mathrm{eV})$ films. Spectra are averaged over the indicated pump-probe delays. The pump fluence is $30 \mu \mathrm{Jcm}^{-2}$ for the first pass and $0.4 \mu \mathrm{Jcm}^{-2}$ for the last pass. The TA data can be numerically decomposed into 3 components using a genetic algorithm (GA). The extracted spectra and kinetics of the components are shown in (b) and (c) in solid lines. Comparison to measured data (dashed) indicates that the blue component corresponds to Pc, the red component to the initial PbSe high-energy (HE) state, the black component to relaxed PbSe low-energy (LE) state. The Pc component in $\mathrm{Pc} / \mathrm{PbSe}$ initially decays faster than in the pristine Pc (dashed blue in (c)) due to triplet transfer, and then revives due to hole transfer from PbSe into Pc. This charge transfer is accompanied by a shift of a PbSe GSB due to electroabsorption (pink) shown in (d). 

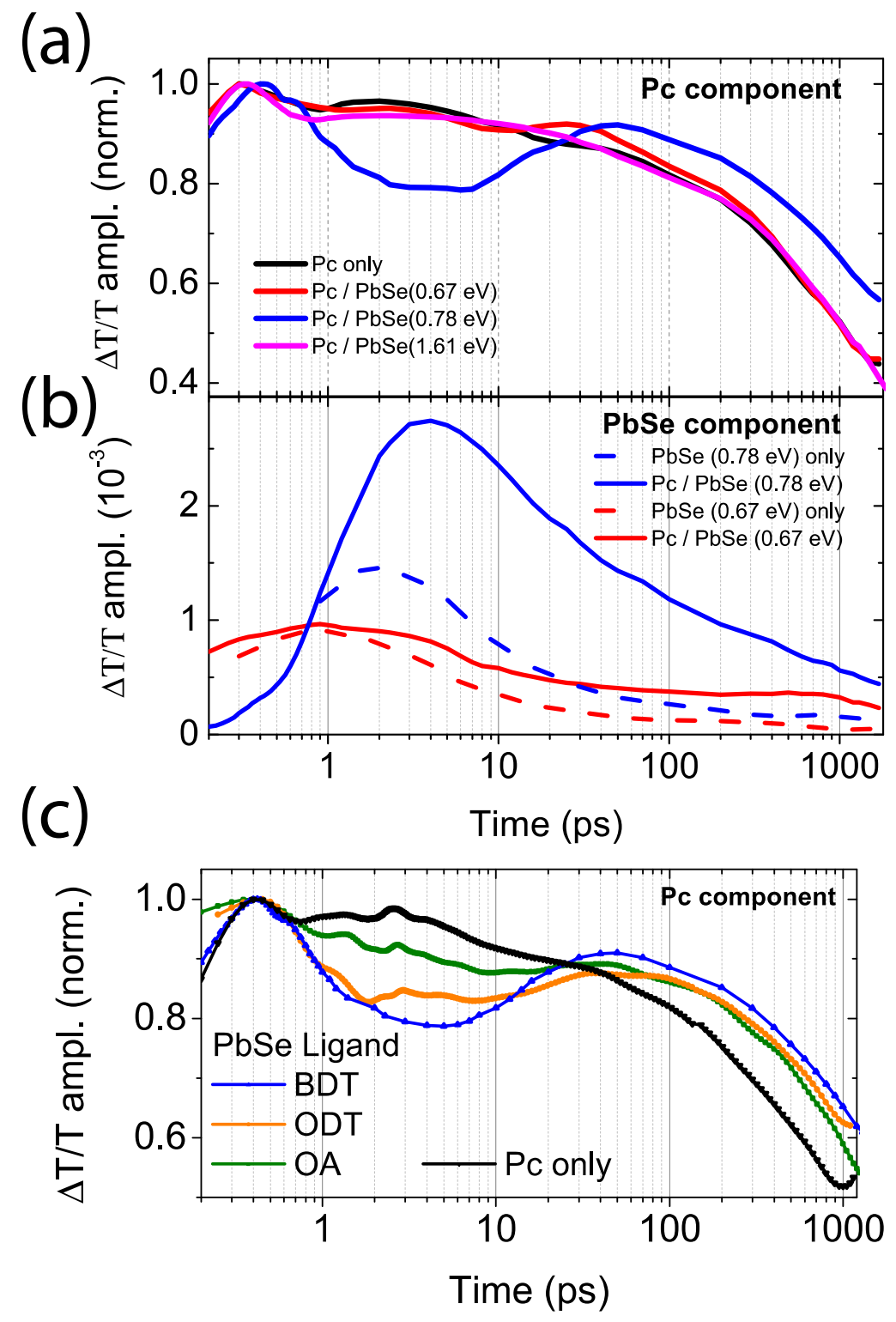

Figure 3: Kinetics of triplet transfer (a) Normalized kinetics of the Pc spectral component extracted via the GA from transient absorption data of $\mathrm{Pc} / \mathrm{PbSe}$ bilayers with varying nanocrystal bandgaps (in brackets). The ligand of the nanocrystals is 1,3-benzenedithiol (BDT). (b) shows the corresponding $\mathrm{PbSe}$ spectral component, comparing pristine nanocrystal films with bilayers with $\mathrm{Pc}$. The $\mathrm{PbSe}$ population in $\mathrm{Pc} / \mathrm{PbSe}(0.78 \mathrm{eV})$ is much larger and peaks at later times compared with the population in pristine $\mathrm{PbSe}(0.78 \mathrm{eV})$, due to triplet transfer (TT). (c) illustrates the evolution of the Pc component in $\mathrm{Pc} / \mathrm{PbSe}$ bilayers with nanocrystal ligands of different length, benzenedithiol (BDT, 0.7nm), octanedithiol (ODT, 1.1nm) and oleic acid (OA, $2.2 \mathrm{~nm}$ ), showing that the triplet transfer efficiency increases for shorter ligands. 
(a)

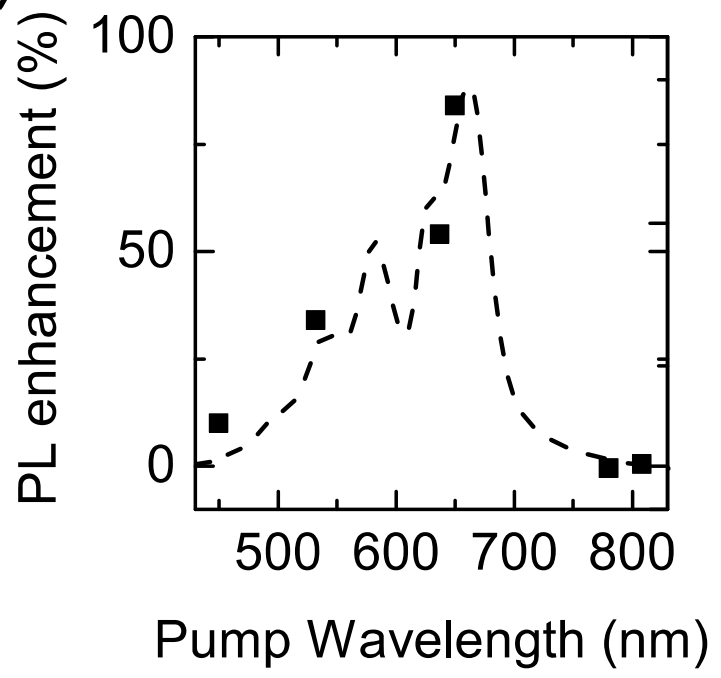

(b)

Pc PbSe

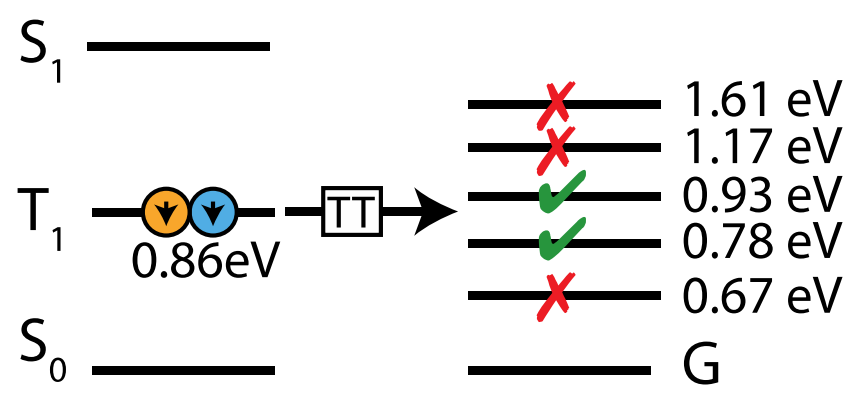

Figure 4: Enhancement of photoluminescence (PL) from PbSe due to triplet transfer. (a) Relative change in PL (squares) for a $\mathrm{Pc} / \mathrm{PbSe}(0.93 \mathrm{eV})$ bilayer in comparison to a $\mathrm{PbSe}(0.93$ $\mathrm{eV}$ ) film. Enhanced PL is only observed for excitation energies higher than the bandgap of Pc and correlates with the absorption of Pc (dashed line). The cw-pump lasers for the PL measurement had an intensity of $100 \mu \mathrm{W} / \mathrm{mm}^{2}$. (b) summarizes for which nanocrystal bandgaps triplet transfer was observed. It shows that triplet transfer from $\mathrm{Pc}$ into $\mathrm{PbSe}$ is efficient for the nanocrystal bandgaps close to resonance with the triplet energy level in Pc. 


\section{Methods}

Nanocrystal fabrication: All chemicals were purchased from Sigma Aldrich, if not stated otherwise, and were anhydrous if available. PbSe nanocrystals were synthesized following standard methods ${ }^{20}$. Briefly, $\mathrm{Pb}(\mathrm{OAc}) 2 \mathrm{H} 2 \mathrm{O}(3.44 \mathrm{mmol} ; 1.3 \mathrm{~g})$, oleic acid (OA; $8.58 \mathrm{mmol} ; 2.7$ $\mathrm{ml})$ and 1-octadecene (ODE; $75 \mathrm{mmol} ; 24 \mathrm{ml})$ were degassed at $100{ }^{\circ} \mathrm{C}$ under vacuum $(10-2$ mbar or better) for $2 \mathrm{~h}$. In order to form the $\mathrm{Pb}$-oleate precursor complex the temperature was raised to $160{ }^{\circ} \mathrm{C}$ under nitrogen atmosphere and subsequently changed to the desired Seprecursor injection temperature $\left(120^{\circ} \mathrm{C}-180^{\circ} \mathrm{C}\right.$ ). In parallel, Se (Alfa Aeser, $10.8 \mathrm{mmol} ; 852.8$ $\mathrm{mg}$ ), diphenylphosphine (DPP; $15 \mu \mathrm{mol} ; 26.1 \mu \mathrm{l}$ ) and tri- $n$-octylphosphine (TOP; $24.2 \mathrm{mmol}$; $10.8 \mathrm{ml}$ ) were combined and stirred under nitrogen atmosphere to form the Se-precursor. PbSe nanocrystal growth was initiated by the rapid injection of the Se-precoursor into the prepared $\mathrm{Pb}$ oleate solution. After the desired nanocrystal size was reached $(20 \mathrm{sec}-5 \mathrm{~min})$ the reaction was quenched by injecting $20 \mathrm{ml}$ of hexane and by placing the flask into an ice-cooled water bath. Subsequent purification steps were carried out in an argon-filled glove box. The nanocrystals were extracted via repeated precipitation with a mixture of 1-butanol and ethanol.

Sample Fabrication: Samples were fabricated on $0.13 \mathrm{~mm}$ thin cover glass slides. Nanocrystal films were deposited by a conventional layer-by-layer method ${ }^{20}$, in an inert environment, using crosslinking ligands (20mM 1,3-benzeneditiol or octanedithiol in acetonitrile), from 2 layers of a $4 \mathrm{mg} / \mathrm{mL}$ solution of $\mathrm{PbSe}$. For films from $\mathrm{PbSe}$ capped with the native oleic acid ligand, a single layer of PbSe was spin coated from $8 \mathrm{mg} / \mathrm{mL}$ solution. Subsequently, $5 \mathrm{~nm}$ of pentacene was evaporated on the nanocrystal films, in a vacuum better than $2 \times 10-6$ mbar. The samples were encapsulated with a second $0.13 \mathrm{~mm}$ thin glass slide and an epoxy glue before exposing to air.

Steady State Optical Measurements: The absorption spectra of the nanocrystals were taken in solution at $0.05-1 \mathrm{mg} / \mathrm{mL}$ using a PerkinElmer Lambda $9 \mathrm{UV}$ - Vis-IR spectrophotometer. PL was measured by illuminating a spot of ca. $2 \mathrm{~mm}$ in diameter with a diode lasers (LDM405.100.CWA.L for $405 \mathrm{~nm}$, Thorlabs CPS450 for 450nm, MGL-III-532 for $532 \mathrm{~nm}$, Thorlabs CPS635S for $637 \mathrm{~nm}$, SMFR-R0004 for $650 \mathrm{~nm}$, Lasermax-MDL for $780 \mathrm{~nm}$, IQu1C135 for $808 \mathrm{~nm}$ ). Lenses project the PL emitted to a solid angle of $0.1 \pi$ onto an InGaAs detector (Andor DU490A-1.7) which has a cut-off at $1600 \mathrm{~nm}$. For PL measurements in a magnetic field, we used an electromagnet from GMW Model 3470 with $1 \mathrm{~cm}$ distance between cylindrical poles. The samples were tilted around the vertical axis so that the pump was incident (and the PL was measured) at an angle of $60^{\circ}$.

Transient absorption (TA) spectroscopy: In this technique a pump pulse generates photoexcitations within the film, which are then studied at some later time using a broadband probe pulse. A portion of the output of a Ti:Sapphire amplifier system (Spectra-Physics Solstice) operating at $1 \mathrm{KHz}$, was used to pump a TOPAS optical parametric amplifier (Light Conversion), to generate narrowband (10nm FWHM) pump pulses centered at 550nm. Another portion of the amplifier output was used to pump a home built non-collinear optical parametric 
amplifier (NOPA). The probe beam was split to generate a reference beam so that laser fluctuations could be normalized. Pump and probe beams were made collinear with a beam splitter and entered an optical cavity, based on the design on Kandada et al. ${ }^{21}$, consisting of two concave mirrors (focal length $\mathrm{f}$ ) placed $4 \mathrm{f}$ apart from each other with the sample in the center. A schematic is shown in $\mathrm{S} 1$. The beams underwent multiple bounces in the cavity, making multiple passes in the sample, thus allowing for the weak signal from the thin layers to be amplified. After exiting the cavity a long pass filter was used to block the pump beam, while allowing the probe beam to pass. The probe and reference beams were dispersed in a spectrometer (Andor, Shamrock SR-303i) and detected using a pair of 16-bit 512-pixel linear image sensors (Hamamatsu). The probe was delayed using a mechanical delay stage (Newport) and every second pump pulse was omitted using a mechanical chopper. Data acquisition at $1 \mathrm{kHz}$ was enabled by a custom-built board from Stresing Entwicklunsbüro. The differential transmission $(\Delta \mathrm{T} / \mathrm{T})$ was calculated after accumulating and averaging 1000 "pump on" and "pump off" shots for each data point.

As discussed by Kandada et al. ${ }^{21}$, due to the group velocity mismatch between pump and probe wavelengths there is a reduction in time resolution of the experiment. From the rise time of the signal we estimate the time resolution of the experiment to be about $300 \mathrm{fs}$ at $670 \mathrm{~nm}$. While this is insufficient to study the initial singlet fission process in Pc, which proceeds on sub $100 \mathrm{fs}$ timescales, it is sufficient to study the triplet transfer process.

Numerical Methods: We use numerical methods based on a genetic algorithm to deconvolute the overlapping spectral signatures of individual excited states and obtain their kinetics. The full details of this approach can be found elsewhere ${ }^{23,24}$. In summary, a large population of random spectra are generating and bred to form successive generations of offspring, using a survival of the fittest approach. The best spectra are returned as optimized solutions. For a given solution, the fitness is calculated as the inverse of the sum of squared residual with a penalty added for non-physical results. The parent spectra are selected using a tournament method with adaptive crossover. The offspring are generated using a Gaussian-function mask of random parameters 


\section{References}

1. Dexter, D.L. Two ideas on energy transfer phenomena: Ion-pair effects involving the OH stretching mode, and sensitization of photovoltaic cells. Journal of Luminescence (1979).

2. Qiang, Z. et al. Highly efficient resonant coupling of optical excitations in hybrid organic/inorganic semiconductor nanostructures. Nature nanotechnology (2007).

3. Blumstengel, S., Sadofev, S., Xu, C., Puls, J. \& Henneberger, F. Converting Wannier into Frenkel excitons in an inorganic/organic hybrid semiconductor nanostructure. Physical review letters 97, 237401 (2006).

4. Agranovich, V., Gartstein, Y. \& Litinskaya, M. Hybrid resonant organic-inorganic nanostructures for optoelectronic applications. Chemical Reviews 111, 5179-5214 (2011).

5. Walker, B.J., Bulović, V. \& Bawendi, M.G. Quantum Dot/J-Aggregate Blended Films for Light Harvesting and Energy Transfer. Nano Letters 10, 3995-3999 (2010).

6. Heliotis, G. et al. Hybrid Inorganic/Organic Semiconductor Heterostructures with Efficient Non-Radiative Energy Transfer. Advanced Materials 18, 334-338 (2006).

7. Medintz, I.L. et al. Self-assembled nanoscale biosensors based on quantum dot FRET donors. Nat Mater 2, 630-638 (2003).

8. Carles, C., Alberto, F., Alex, Z. \& Gregory, D.S. Examining Förster Energy Transfer for Semiconductor Nanocrystalline Quantum Dot Donors and Acceptors. The Journal of Physical Chemistry C (2008).

9. Scholes, G.D. Long-range resonance energy transfer in molecular systems. Annual review of physical chemistry 54, 57-87 (2003).

10. Köhler, A. \& Bässler, H. Triplet states in organic semiconductors. Materials Science and Engineering: R: Reports 66, 71 (2009).

11. Smith, M. \& Michl, J. Singlet fission. Chemical Reviews 110, 6891-6936 (2010).

12. Rao, A., Wilson, M., Albert-Seifried, S., Di Pietro, R. \& Friend, R.H. Photophysics of pentacene thin films: The role of exciton fission and heating effects. Physical Review B 84, 195411 (2011).

13. Wilson, M. et al. Ultrafast dynamics of exciton fission in polycrystalline pentacene. Journal of the American Chemical Society 133, 11830 (2011).

14. Shockley, W. \& Queisser, H.J. Detailed Balance Limit of Efficiency of p-n Junction Solar Cells. Journal of Applied Physics 32(1961).

15. Lee, J. et al. Singlet Exciton Fission Photovoltaics. Accounts of Chemical Research 46, 1300-1311 (2013).

16. Wilson, M., Rao, A., Ehrler, B. \& Friend, R. Singlet Exciton Fission in Polycrystalline Pentacene: From Photophysics toward Devices. Accounts of Chemical Research 46, 1330 (2013).

17. Rao, A. et al. Exciton fission and charge generation via triplet excitons in pentacene/C60 bilayers. Journal of the American Chemical Society 132, 12698 (2010).

18. Congreve, D. et al. External quantum efficiency above $100 \%$ in a singlet-exciton-fission-based organic photovoltaic cell. Science 340, 334-337 (2013).

19. Thompson, N.J., Congreve, D.N., Goldberg, D., Menon, V.M. \& Baldo, M.A. Slow light enhanced singlet exciton fission solar cells with a 126\% yield of electrons per photon. Applied Physics Letters 103, - (2013).

20. Ehrler, B. et al. In situ measurement of exciton energy in hybrid singlet-fission solar cells. Nature Communications 3, 1019 (2012).

21. Kandada, A. et al. Ultrafast energy transfer in ultrathin organic donor/acceptor blend. Scientific reports $\mathbf{3}$, 2073 (2013).

22. Geacintov, N.E., Burgos, J., Pope, M. \& Strom, C. Heterofission of pentacene excited singlets in pentacene-doped tetracene crystals. Chemical Physics Letters 11, 504-508 (1971).

23. Rao, A. et al. The role of spin in the kinetic control of recombination in organic photovoltaics. Nature 500, 435-439 (2013).

24. Gélinas, S. et al. Ultrafast long-range charge separation in organic semiconductor photovoltaic diodes. Science 343, 512-516 (2014).

25. Gdor, I. et al. Exploring exciton relaxation and multiexciton generation in $\mathrm{PbSe}$ nanocrystals using hyperspectral near-IR probing. ACS nano 6, 3269-3277 (2012).

26. Liu, X., Iimori, T., Ohshima, R., Nakabayashi, T. \& Ohta, N. Electroabsorption spectra of PbSe nanocrystal quantum dots. Applied Physics Letters 98, - (2011).

27. Dexter, D.L. A Theory of Sensitized Luminescence in Solids. The Journal of Chemical Physics (1953). 
28. Kang, I. \& Wise, F.W. Electronic structure and optical properties of PbS and PbSe quantum dots. Journal of the Optical Society of America B 14, 1632-1646 (1997).

29. Tischler, J.G. et al. Band-edge excitons in PbSe nanocrystals and nanorods. Physical Review B 82, 245303 (2010).

30. Thompson, N.J. et al. Nanostructured Singlet Fission Photovoltaics Subject to Triplet-Charge Annihilation. Advanced Materials 26, 1366-1371 (2014).

Acknowledgements: M.T. thanks the Gates Cambridge Trust for funding. B.E. thanks the KACST-Cambridge Research Project for support and Selwyn College, Cambridge for a Research Fellowship. B.J.W was supported by a Herchel Smith Research Fellowship. M.L.B. was supported by Studienstiftung des Deutschen Volkes. A.R. thanks Corpus Christi College, Cambridge for a Research Fellowship. This work was supported by the EPSRC and the Winton Programme for the Physics of Sustainability.

Author Contributions: M.T prepared samples, performed time resolved and steady state optical measurements, analyzed the data and wrote the paper. B.E prepared and characterized the samples and wrote the paper. S.G setup the multi-pass experiments. M.L.B synthesized the nanocrystals. B.J.W performed steady state optical measurements. K.P.M characterized the samples. N.C.G supervised the work of M.L.B. R.H.F supervised the work of M.T, S.G and K.P.M and wrote the paper. A.R initiated and guided the work, setup the multi-pass experiments, performed time resolved optical measurements and wrote the paper.

Author Information: Correspondence and requests for materials should be addressed to Akshay Rao (ar525@cam.ac.uk). 